\title{
New Mathematical Modelling of Blood Lactate Kinetics during Ramp Mode Exercise in Man
}

\author{
Yoshiyuki Fukuba, Sachio Usui,* and Masaki Munaka \\ Department of Biometrics, Research Institute for Nuclear Medicine \\ and Biology, Hiroshima University, Minami-ku, Hiroshima, 734 Japan \\ * Laboratory for Exercise Physiology and Biomechanics, \\ Faculty of Integrated Arts and Sciences, \\ Hiroshima University, Naka-ku, Hiroshima, 730 Japan
}

\begin{abstract}
Summary The lactate (LA) kinetics of the whole body in exercise was described using the two-compartment model in consideration of the underlying physiological mechanism, and LA kinetics during the ramp mode exercise was analyzed. The estimated pattern of the glycolytic energy supply showed the progressive increase concurrently with the onset of ramp mode exercise and this pattern was just like a ramp phenomenon. Based on this result, it is proposed that blood LA kinetics during ramp exercise should be described mathematically as a continuous model, called "2nd-order model."
\end{abstract}

Key words: lactate kinetics, ramp exercise, two-compartment model.

The kinetics of glycolytic energy supply (lactate (LA) metabolism) during exercise have been studied generally from the measurements of LA concentration in blood. Although this measurement is of practical value, it is insufficient for the quantitative and qualitative evaluation of the kinetics in real LA production. This is because blood LA kinetics were found to result from the dynamic equilibrium regarding production, removal (oxidation and excretion), and exchange (uptake and release) of LA, according to recent studies by an isotopic tracer technique (e.g., STANLEY et al., 1985), the muscle biopsy technique (e.g., KARLsSON, 1970), the MRspectrographic analysis (e.g., DAwson et al., 1978), and the mathematical models to LA kinetics in the whole body (e.g., Zouloumian and Freund, 1981a).

There has been a controversy about the mathematical expression of blood LA kinetics during ramp mode exercise (Hughson et al., 1987). The model is divided into two types. One is the "threshold model" (BEAVER et al., 1985), and the other is the "continuous model" (Hughson et al., 1987). The main point of their discussion

Received for publication Februry 6, 1989 
(HUGHSON et al., 1987) is to examine only the goodness of fit of each model, that is, the value of the mean square error and the systematic bias of the residual. Either proposed model does not seem to be based on some physiological mechanism. Therefore, our purpose is to propose a new mathematical expression of blood LA kinetics during ramp mode exercise on the basis of the model analysis in consideration of some underlying physiological mechanism. For this purpose, we analyzed LA kinetics with the following procedures: 1) the formulation of LA kinetics by the two-compartment model and its solution; 2) the estimation of the pattern in real LA production from blood LA kinetics during ramp mode exercise using the deconvolution technique; 3) the proposal of a new mathematical expression of blood LA kinetics during ramp mode exercise.

\section{Two-compartment model for LA kinetics}

Freund and his colleagues (ZOUlOUMIAN and FreUnd, 1981a, b) constructed and mathematically solved a two-compartment model that can satisfactorily account for the observed LA kinetics after an intense step exercise. The model is based on the law of mass conservation in a volume $(V)$. It allows for LA production and utilization, and for LA uptake from other compartments and LA release to other compartments. If the time-serial concentration of LA is denoted by LA $(t)$, the volume of LA in each compartment at time $t$ can be expressed by

$$
V_{\mathrm{i}} \cdot \frac{\mathrm{d}}{\mathrm{d} t} \mathrm{LA}_{\mathrm{i}}(t)=\mathrm{LP}+\mathrm{LU}-\mathrm{LR}-\mathrm{LUT},
$$

where LP, LA production; LU, LA uptake; LR, LA release; LUT, LA utilization; i, muscle or space.

Definition of the model. A diagram of the model used in this study is shown in Fig. 1a. The fundamental assumptions of the model are as follows:

1) The total LA distribution space is composed of two compartments: the working muscle (M) and the remaining LA space (S), having respectively the constant volumes $V_{\mathrm{M}}$ and $V_{\mathrm{S}}$ in which the time-serial concentrations $\mathrm{LA}_{\mathrm{M}}(t)$ and $\operatorname{LA}_{\mathrm{S}}(t)$ prevail.

2) The rates of LA release, uptake, and utilization in $M$ and $S$ are proportional to the LA contents of these compartments; i.e., $V_{\mathrm{M}} \cdot \mathrm{LA}_{\mathrm{M}}(t)$ and $V_{\mathrm{S}} \cdot \mathrm{LA}_{\mathrm{S}}(t)$. The coefficients of proportionality in all cases are positive constants.

3) The real LA production rate in $\mathbf{M}$ is the unknown time-varying variable.

4) LA concentration in arterial or venous blood represents the average value in $\mathrm{S}$, i.e., $\mathrm{LA}_{\mathrm{S}}(t) \simeq$ blood $\mathrm{LA}(t)$.

The validity and limitation of a great part of these assumptions are already discussed by Zouloumian and Freund (1981a,b) and MORTON (1984). Additionally, to simplify the analysis, we assumed that,

5) LA production in S can be ignored, because LA production in other tissue is relatively small as compared with that in the active muscle during exercise. 
(a)

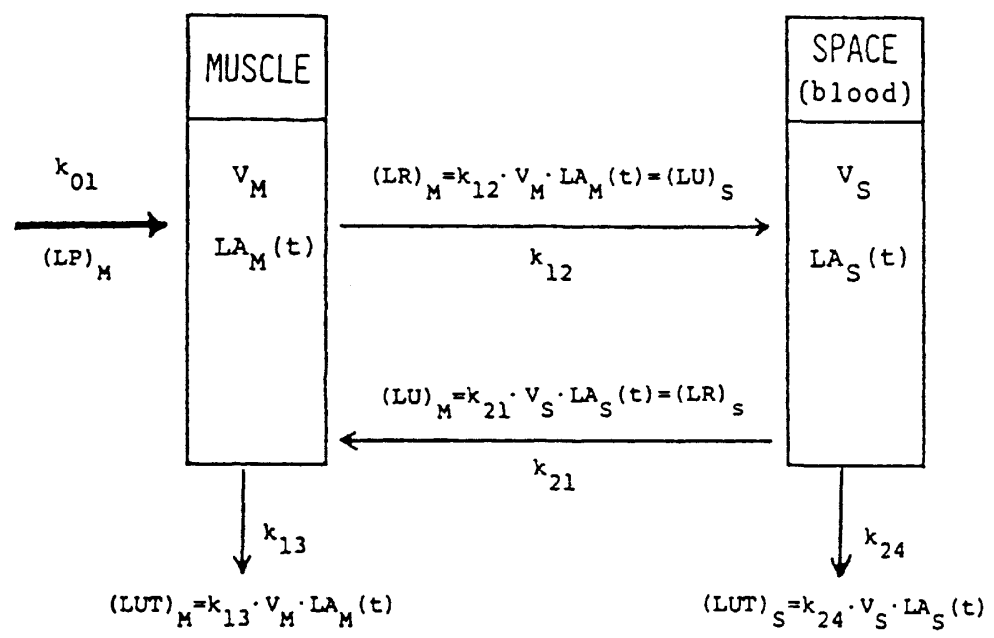

(b)

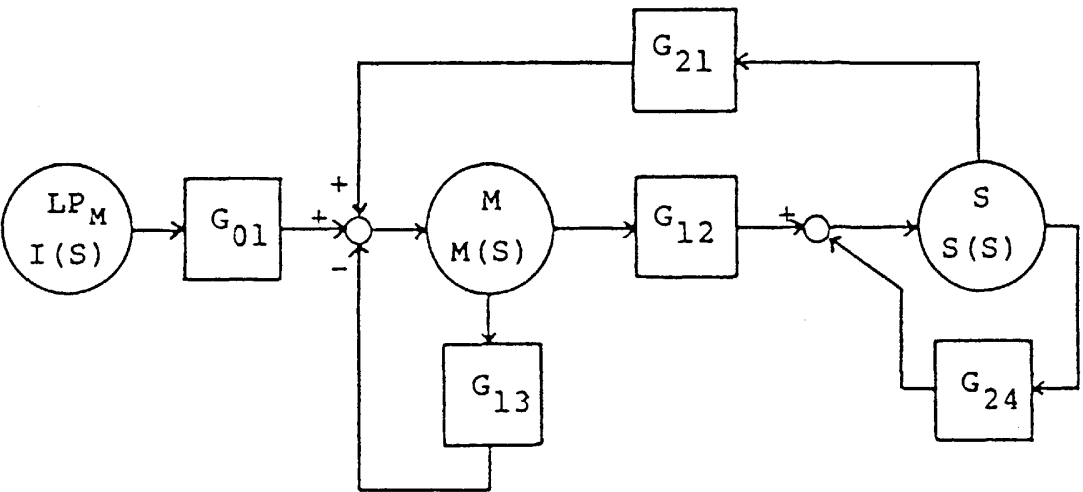

Fig. 1. Structure of the two-compartment model (a) and its block diagram (b) for LA kinetics in exercise.

Formulation and its solution. Figure $1 \mathrm{~b}$ shows a block diagram of the twocompartment model for the LA kinetics described above. We deal with "excess LA" above the resting level hereafter. Assuming zero initial conditions, from the input $I(s)$ (= real LA production in active muscle), the output $S(s)$ (=space LA) can be expressed by a Laplace transform as follows:

$$
S(s)=\frac{G_{01} * G_{12}}{\left(1+G_{24}\right) *\left(1+G_{13}\right)-G_{12} * G_{21}} * I(s),
$$

where $G_{i j}=k_{i j} / s$.

The transfer functions among real LA production in active muscle, muscle LA, and space (blood) LA can be expressed respectively as shown in Table 1. Since the system always has the non-oscillatory damped mode, $g(t)$ of each transfer function, 


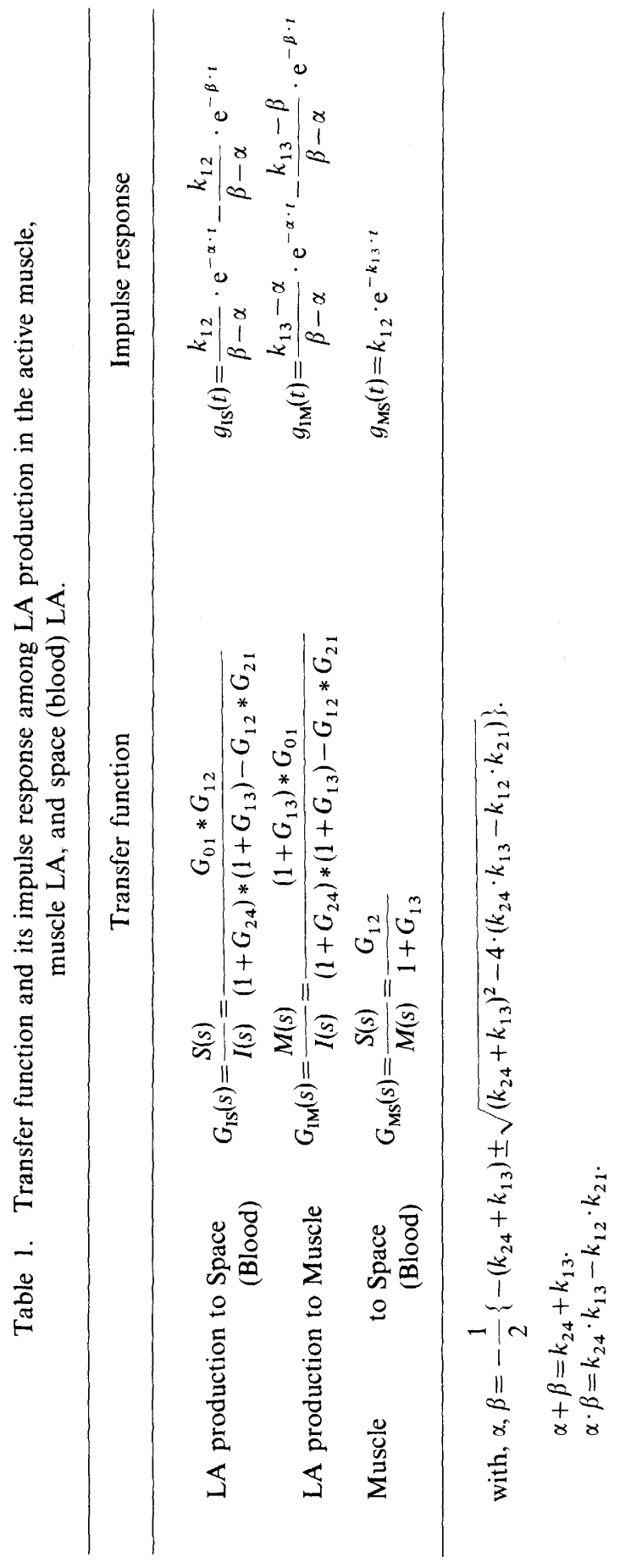


which was obtained from the inverse Laplace transform, i.e., impulse response, can be expressed by the combinations of exponential terms of time $t$ also shown in Table 1.

\section{Procedure of analysis}

Estimation of parameters in the model. Impulse, step, ramp, and sine wave are commonly used for the mode of input to identify the system. The input of the system in this study is the real LA production above the resting level so that it is difficult to set the mode of input which satisfies our request. The acceptable mode for assumption is the step-off input of real LA production which is expected to occur after the high intensity step input exercise. Now, we consider the step-on and -off input, i.e., the onset of a constant load exercise and its recovery phase. When the input is expressed as $I(s)=K / s$ ( $K$, gain of input), the output $\operatorname{LA}_{\mathrm{S}}(t)$ during the constant load exercise can be expressed as,

$$
\mathrm{LA}_{\mathrm{S}}(t)=K \cdot\left(\frac{k_{12}}{\alpha \cdot \beta}+\frac{k_{12}}{\alpha \cdot(\alpha-\beta)} \cdot \mathrm{e}^{-\alpha \cdot t}-\frac{k_{12}}{\beta \cdot(\alpha-\beta)} \cdot \mathrm{e}^{-\beta \cdot t}\right), \quad 0 \leq t<\theta
$$

where $t=0$, the onset of exercise, and $\theta$, the duration of exercise.

In case the step exercise is stopped before $\operatorname{LA}_{S}(t)$ reaches the plateau level (it happens in most cases of actual high intensity step exercise), $\operatorname{LA}_{\mathbf{S}}(t)$ in its recovery phase is expressed as,

$$
\begin{aligned}
& \qquad \operatorname{LA}_{\mathrm{S}}(T)=K \cdot\left\{\frac{-k_{12}}{\alpha \cdot(\alpha-\beta)} \cdot\left(1-\mathrm{e}^{-\alpha \cdot \theta}\right) \cdot \mathrm{e}^{-\alpha \cdot T}+\frac{k_{12}}{\beta \cdot(\alpha-\beta)} \cdot\left(1-\mathrm{e}^{-\beta \cdot \theta}\right) \cdot \mathrm{e}^{-\beta \cdot T}\right\} \\
& =A \cdot \mathrm{e}^{-\alpha \cdot T}+B \cdot \mathrm{e}^{-\beta \cdot T}, \quad 0 \leq T<\infty \\
& \text { with } \quad A=\frac{-K \cdot k_{12}}{\alpha \cdot(\alpha-\beta)} \cdot\left(1-\mathrm{e}^{-\alpha \cdot \theta}\right), \quad B=\frac{K \cdot k_{12}}{\beta \cdot(\alpha-\beta)} \cdot\left(1-\mathrm{e}^{-\beta \cdot \theta}\right)
\end{aligned}
$$

where $T(=t-\theta)=0$, the onset of the recovery.

The feature of this equation is that it is consistent with the blood LA kinetics curve in the recovery phase observed in many previous studies. As Eqs. (2) and (3) indicate, $k_{12}$ cannot be estimated definitely, but $\alpha$ and $\beta$ can be estimated. Then, we estimate $A, B, \alpha$, and $\beta$ in Eq. (3) using the non-linear least squares method from the data set of the observed blood LA in the recovery phase after the high intensity step exercise. Here, we used the results of the study by Freund and Zouloumian (1981a). They observed blood LA kinetics during 60-min recovery after the bicycle ergometer exercise in 3-min step input at the work load levels of 76, 90, and $100 \%$ of $\dot{V}_{\mathrm{O}_{2} \max }$. We recalculated their results and adopted $\hat{A}=-10.69, \hat{B}=15.42, \hat{\alpha}=0.480$, and $\hat{\beta}=0.0723$ during the recovery from the step exercise of $100 \%$ of $\dot{V}_{\mathrm{O}_{2} \max }$. If $k_{12}$ is assumed to be a plausible given value, $g_{\mathrm{IS}}(t)$, which is the impulse response of the system of input $I(s)$ and output $S(s)$, can be expressed using $\hat{\alpha}$ and $\hat{\beta}$ concretely.

Experiment. Thirteen young male students volunteered as the subjects of this study. Their mean age, height, and weight were 21 years, $168 \mathrm{~cm}$, and $59 \mathrm{~kg}$, 
respectively. Subjects were informed of the nature, purpose, and possible risks involved in the study before giving their voluntary consent to participate. They performed a progressive incremental work rate exercise (just like a ramp mode exercise) on a Monark cycle ergometer. After a 10-min rest, each subject carried out the exercise test, in which the work rate was increased by $150 \mathrm{kgm}$ every $2 \mathrm{~min}$ from $0 \mathrm{kgm}$ ( = unloaded pedalling) up to his limit of volitional fatigue. The venous blood sample was taken from a warmed ear lobe at rest and during the last $30 \mathrm{~s}$ of each work rate and was analyzed for LA concentration using the enzymatic method.

Estimation of the pattern in real LA production. In general, the impulse response $g(t)$, input $x(t)$, and output $y(t)$ in the linear system can be expressed as the form of convolution as follows:

$$
y(t)=\int_{0}^{t} g(\lambda) \cdot x(t-\lambda) \mathrm{d} \lambda=\int_{0}^{t} g(t-\lambda) \cdot x(\lambda) \mathrm{d} \lambda,
$$

where $\lambda$ is a dummy variable. In the system which is dealt with here, $g(t)$ is determined by giving a concrete value to $k_{12}$, and the output $y(t)$ is measured in the experiment. By the deconvolution that is the inverse procedure of the convolution, the unknown input $x(t)$ is calculated from $g(t)$ and $y(t)$ theoretically. The numerical calculation of the deconvolution, however, cannot be successfully executed if $y(t)$ contains the serious noise. Therefore, before executing the deconvolution, we smoothed the observed output $\mathrm{LA}_{\mathrm{S}}(t)$ using the non-parametric smoothing technique LOWESS (CLEVELAND, 1979). We estimated the time-serial LA production rate by the deconvolution of $g_{\mathrm{IS}}(t)$ and the smoothed $\operatorname{LA}_{\mathrm{S}}(t)$. A major focus was whether the pattern in real LA production during the ramp mode exercise is like a ramp or is like a threshold when LA kinetics are assumed to be described by the two-compartment model. Then we gave the plausible value to $k_{12}$ and $V_{\mathrm{S}}\left(k_{12}=0.2\right.$ $\left(\mathrm{min}^{-1}\right)$ and $V_{\mathrm{S}}=26(l)$, determined by referring to the studies of FREUND and Zouloumian (1981b), and HeCK et al. (1985)), and we paid attention only to the pattern of the relative change in time-serial real LA production estimated by the deconvolution. As a result, in most of the 13 subjects, the estimated pattern in real LA production showed an increase like a ramp phenomenon (Fig. 2). It was thus considered that the glycolytic energy during the ramp exercise was supplied by the ramp pattern.

Proposal of a new mathematical description of blood LA kinetics during ramp exercise

According to the result described above, we proposed a new mathematical modelling of blood LA kinetics during ramp exercise in man. If the pattern of supply in glycolysis is assumed to be a ramp, i.e., $I(s)=a / s^{2}$ ( $a$, gain of input), the blood LA kinetics as the output $\operatorname{LA}_{\mathbf{S}}(t)$ can be expressed as

$$
\operatorname{LA}_{\mathbf{s}}(t)=a \cdot k_{12} \cdot\left\{t-\frac{\beta^{2}}{\alpha \cdot \beta \cdot(\beta-\alpha)} \cdot\left(1-\mathrm{e}^{-\alpha \cdot t}\right)+\frac{\alpha^{2}}{\alpha \cdot \beta \cdot(\beta-\alpha)} \cdot\left(1-\mathrm{e}^{-\beta \cdot t}\right)\right\},
$$

and actual blood LA concentration $\operatorname{LA}_{\mathrm{b}}(t)$ is expressed as 


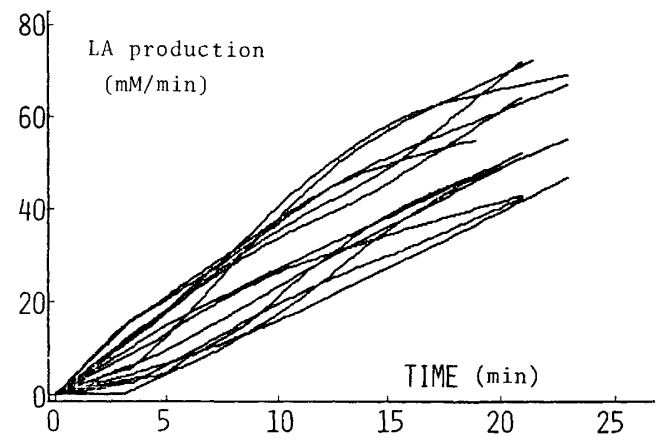

Fig. 2. Estimated pattern in real LA production of each subject based on the twocompartment model.

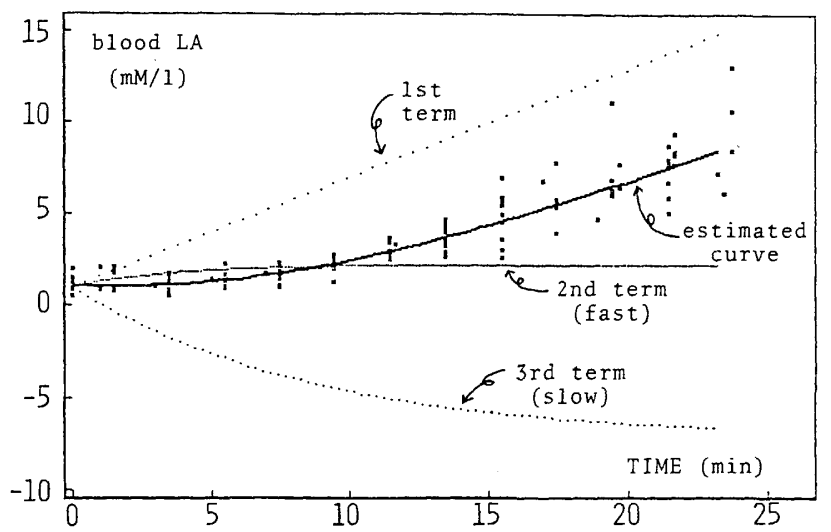

Fig. 3. Graphical representation of the estimated results in each term of " 2 nd-order model" (Eq. (5)) using the whole data of 13 subjects. Each curve of the 1st, 2nd, and 3rd term in Eq. (5) shows the adjusted one by $\mathbf{L A}_{(\text {rest })}$ for easy visual understanding. Each curve in this figure represents the numerical value of each term in Eq. (5) plus $\mathrm{LA}_{\text {(rest) }}$.

$$
\begin{aligned}
\mathrm{LA}_{\mathrm{b}}(t)= & a \cdot k_{12} \cdot\left\{t-\frac{\beta^{2}}{\alpha \cdot \beta \cdot(\beta-\alpha)} \cdot\left(1-\mathrm{e}^{-\alpha \cdot t}\right)+\frac{\alpha^{2}}{\alpha \cdot \beta \cdot(\beta-\alpha)} \cdot\left(1-\mathrm{e}^{-\beta \cdot t}\right)\right\}+\mathrm{LA}_{(\text {rest })} \\
= & a \cdot k_{12} \cdot t-a \cdot k_{12} \cdot \frac{\beta^{2}}{\alpha \cdot \beta \cdot(\beta-\alpha)} \cdot\left(1-\mathrm{e}^{-\alpha \cdot t}\right)+a \cdot k_{12} \cdot \frac{\alpha^{2}}{\alpha \cdot \beta \cdot(\beta-\alpha)} \cdot\left(1-\mathrm{e}^{-\beta \cdot t}\right) \\
& +\mathrm{LA}_{(\text {rest })}
\end{aligned}
$$

We called this expression " 2 nd-order model." The estimation of the parameters in Eq. (5) was tried with the pooled data of 13 subjects using the weighted non-linear least squares method. The estimates of $a \cdot k_{12}, \alpha, \beta$, and the resting level of blood LA, i.e., $\mathrm{LA}_{\text {(rest) }}$, were $0.63,0.30$ (time constant $\fallingdotseq 3.3 \mathrm{~min}$ ), 0.11 (time constant $\fallingdotseq 9.1$ 
$\min$ ), and 1.07, respectively, and the estimated curve of $\operatorname{LA}_{b}(t)$ is shown in Fig. 3. Compared with $\hat{\alpha}$ and $\hat{\beta}$ in the recovery phase, $\hat{\beta}$ of the ramp exercise was not in the same order. Further research will be necessary to examine whether the cause of this difference depends on merely the difference of the experimental conditions and the subjects, or depends on the non-linearity of the system.

\section{Additional comments}

Reviewing the recent methodological studies on anaerobic threshold (AT), the mathematical expression of the blood LA kinetics during the ramp mode exercise is divided into two types. One is the "threshold model" proposed by the Wasserman group. The original model was the one in which the regression relation of LA to time (or $\dot{V}_{\mathrm{O}_{2}}$ ) was expressed as 2-segment linear regression model (GREEN et al., 1983). Thereafter, the improved model, in which the 2 -segment linear regression was applied to the data set expressed not as the normal scale but as the log-log scale, was proposed (BEAver et al., 1985; LundBerG et al., 1986). The "threshold model" is based on the following assumption: when the work intensity is increased above AT, the acceleration in glycolysis (=threshold phenomenon in real LA production) causes blood LA to elevate; as a result, the blood LA kinetics during the ramp exercise shows a threshold phenomenon.

The other type is the "continuous model." Firstly, YEH et al. (1983) found that the mono-exponential model fits the data set better than the 2-segment linear model. Thereafter, Hughson et al. (1987) modified the model to the exponential plus constant model. They pointed out that the blood LA increased as a continuum and it was detected as a threshold only because of the limitation of current methodology. This controversy is important regarding the physiological basis of AT, that is, regarding whether blood LA kinetics behaves as a threshold phenomenon or not. Nevertheless, either mathematical modelling does not seem to be based on the plausible physiological mechanism. The result in this study indirectly supports the view of the continuous type's group. It has been pointed out repeatedly by the continuous type's group, that the blood LA kinetics during ramp mode exercise is only a result of the dynamic equilibrium of the appearance and disappearance of LA (BROOKS, 1985).

It is well known that there is a forearm arterio-venous LA difference during ramp mode exercise of the leg muscles (Yoshide et al., 1982). That is, the LA in venous blood has a positive time lag in relation to the one in arterial blood due to the removal of LA in inactive muscles. In this study, we could not use the arterial LA data. If the pattern in real LA production is estimated by the arterial LA data instead of the venous one, the pattern will show the progressive increase as a more distinct ramp pattern.

We proposed a new model called "2nd-order model," which is one of the continuous type. Figure 3 shows the estimated time-serial change of each term of the right side of Eq. (5). The LA kinetics during the ramp exercise can be described by two main processes: one is the second term which has a fast velocity constant $\alpha$ 
and describes the appearance of LA into blood; the other is the third term which has a slow velocity constant $\beta$ and describes the disappearance of LA into blood. As stated by FREUND et al. (1986), it is appropriate to consider that the fast velocity constant $\alpha$ indicates the dynamic information on the transport of LA from the working muscles to blood, and the slow velocity constant $\beta$ indicates the capacity to remove LA during exercise by the metabolic and buffering processes. It is significant to express the blood LA kinetics during the ramp exercise to the form of Eq. (5), i.e., 2nd-order model, because each parameter in this equation provides valuable information regarding the glycolytic capacity during exercise. It is too difficult, however, to estimate the parameters in this model stably, because the parameter has a high relevancy to each other.

Finally, the directions for further research include the following: 1) to develop the stable estimation method of parameters in the 2nd-order model; 2) to develop a new measurement technique of blood LA which can be sampled more frequently in order to estimate the parameters in the 2nd-order model with better accuracy; and 3 ) to examine the cause of the differences of $\alpha$ and $\beta$ due to the mode of exercise, e.g., ramp, step-on and -off, from both the experimental and the theoretical viewpoints.

A part of this study was supported by a Grant for Life Science Research from the Uehara Memorial Foundation, in 1985. A part of this study was presented at the Seoul Olympic Scientific Congress, September 9-15, 1988, at Chonan, Korea.

\section{REFERENCES}

Beaver, W. L., Wasserman, K., and Whipp, B. J. (1985) Improved detection of lactate threshold during exercise using a log-log transformation. J. Appl. Physiol., 59: 19361940.

Brook, G. A. (1985) Anaerobic threshold: Review of the concept and directions for future research. Med. Sci. Sports Exercise, 17: 22-31.

Cleveland, W. S. (1979) Robust locally weighted regression and smoothing scatterplots. JASA, 74: 829-836.

Dawson, M. J., Gadian, D. G., and Wilkie, D. R. (1978) Muscular fatigue investigated by phosphorus nuclear magnetic resonance. Nature, 274: 861-866.

Freund, H., Oyono-Enguelle, S., Heitz, A., Marbach, J., Ott, C., Zouloumian, P., and LAMPERT, E. (1986) Work rate-dependent LA kinetics after exercise in humans. $J$. Appl. Physiol., 61: 932-939.

Freund, H. and Zouloumian, P. (1981a) Lactate after exercise in man: I. Evolution kinetics in arterial blood. Eur. J. Appl. Physiol., 46: 121-133.

Freund, H. and Zouloumian, P. (1981b) Lactate after exercise in man: IV. Physiological observations and model predictions. Eur. J. Appl. Physiol., 46: 161-176.

Green, H. J., Hughson, R. L., Orr, G. W., and Ranney, D. A. (1983) Anaerobic threshold, blood lactate, and muscle metabolites in progressive exercise. $J . A p p l$. Physiol., 54: 1032-1038.

Heck, H., Madar, D., Hess, G., Mucke, S., Muller, R., and Hollman, W. (1985) Justification of the 4-mmol/l lactate threshold. Int. J. Sports Med., 6: 117-130.

Hughson, R., Weisiger, K. H., and Swanson, G. D. (1987) Blood lactate concentration

Vol. 39, No. 2, 1989 
increases as a continuous function in progressive exercise. J. Appl. Physiol., 62: 19751981.

KARLSSON, J. (1970) Lactate and phosphagen concentration in working muscles in man. Acta Physiol. Scand. (Suppl.), 358: 1-78.

Lundberg, M. A., Hughion, R. L., Weisiger, K. H., Jones, R. H., and Swanson, G. D. (1986) Computerized estimation of lactate threshold. Comp. Biomed. Res., 19: 481486.

Morton, R. H. (1984) A mathematical and computer simulation model of the running athlete. In: Ph. D. Thesis, Massey, Univ., Palmerston North, New Zealand, pp. 76-84.

Stanley, W. C., Gertz, E. W., Wisneski, J. A., Morris, D. L., Neese, R. A., and Brooks, G. A. (1985) Systemic lactate kinetics during graded exercise in man. Am.J. Physiol. (Endocrinol. Metabol., 12), 249: E595-E602.

Yeh, M. P., Gardner, R. M., Adams, T. D., Yanowitz, F. G., and Grapo, R. O. (1983) "Anaerobic threshold": Problems of determination and validition. J. Appl. Physiol., 55: 1178-1186.

Yoshida, T., Takeuchi, N., and Suda, Y. (1982) Arterial versus venous blood lactate increase in the forearm during incremental bicycle exercise. Eur. J. Appl. Physiol., 50: 87-93.

Zouloumian, P. and Freund, H. (1981a) Lactate after exercise in man: II. Mathematical model. Eur. J. Appl. Physiol., 46: 135-147.

Zouloumian, P. and Freund, H. (1981b) Lactate after exercise in man: III. Properties of the compartment model. Eur. J. Appl. Physiol., 46: 149-160. 